\title{
¿Efectos Limitados o Poderosos? Una Revisión a la Investigación sobre los Efectos de las Comunicaciones de Campaña en México
}

\section{Limited or Powerful Effects? A Review of Research on the Effects of Campaign Communications in Mexico}

\section{Oniel Francisco Díaz Jiménez Igor Vivero Ávila}

\section{Resumen}

La investigación sobre los efectos de la comunicación política en México ha experimentado un importante avance durante las últimas décadas. Diversos trabajos desde la ciencia política y los estudios en comunicación de masas han realizado aportaciones significativas a la literatura acerca de los efectos de los medios y las campańas electorales en nuevas democracias, con México como estudio de caso. Este artículo ofrece una revisión de los hallazgos de diversos estudios sobre los efectos de las comunicaciones de campańa en elecciones presidenciales sobre el aprendizaje político, la cultura política y el comportamiento político de los mexicanos, señalando también aquellas áreas en las cuales la investigación es aún escasa.

\section{Palabras clave}

Campañas Electorales; Comunicaciones de Campaña; Efectos de las Campañas y de los Medios; Comportamiento Político.

\begin{abstract}
Research on the effects of political communications in Mexico has experienced a significant progress over the past decades. Several studies from political science and communication studies have made significant contributions to the literature on media and campaigns effects in new democracies, with Mexico as a case study. This paper explores the findings of a number of studies on the effects of campaign communications in presidential elections on political learning, political culture and political behavior of Mexicans, also pointing out areas in which research is still scarce.
\end{abstract}

\section{Keywords}

Election Campaigns; Campaign Communications; Campaign and Media Effects; Political Behavior. 


\section{Introducción}

Las elecciones libres y competitivas son una institución fundamental de las democracias contemporáneas. A su vez, las campañas electorales son un componente central de las elecciones democráticas. Una campaña electoral es "el proceso por medio del cual una organización de campaña (ya sea un partido político, candidato o grupo de interés) busca maximizar su rendimiento electoral" (FARRELL, 1996, p. 161). Dicho proceso incluye un conjunto organizado de operaciones de comunicación con el fin de "informar, persuadir y movilizar [al electorado]" (NORRIS, 2002, p. 127). Las campañas cumplen diversas funciones relevantes para la competencia política y pueden movilizar a los ciudadanos para que participen en el proceso electoral de diversas formas; por ejemplo, al buscar noticias sobre las campañas en los medios, al discutir con sus familiares o amigos sobre los temas de las campañas, al asistir a eventos organizados por los equipos de campaña de los candidatos, al donar dinero para financiar alguna campaña o, incluso, al unirse como activistas a la campaña de algún candidato. Las campañas también pueden hacer más (o menos) probable que los ciudadanos voten el día de la elección. Asimismo, los mensajes de las campañas pueden incidir de manera significativa en el comportamiento electoral, funcionando la mayor parte de las veces como mecanismos de activación y/o reforzamiento de las predisposiciones políticas latentes de los votantes; además, en algunas ocasiones funcionan también como instrumentos de persuasión.

Con el fin de sistematizar el estudio académico de las campañas, Pippa Norris (2002) propone un modelo analítico de cuatro elementos: 1) el ambiente contextual conformado por el marco regulatorio, la estructura del sistema de medios de comunicación y del sistema de partidos dentro de cada país; 2) las organizaciones de campaña con los objetivos estratégicos que estas organizaciones intentan comunicar al electorado; 3) los canales de comunicación empleados por estas organizaciones para transmitir sus mensajes políticos, y 4) los efectos de estos mensajes en los públicos objetivo (véase la Gráfica 1$)^{1}$.

\footnotetext{
${ }^{1}$ En función de la efectividad de las campañas, se puede estudiar un quinto elemento, conocido como "circuito de retroalimentación dinámica" (NORRIS, 2002, p. 127), relativo a la forma en que las organizaciones aprenden de las respuestas de su público objetivo y ajustan sus objetivos y estrategias en consecuencia.
} 
Gráfico 1 - Modelo de comunicaciones de campaña y su ambiente contextual

1) Ambiente contextual

Marco regulatorio

2) Organizaciónes de campańa

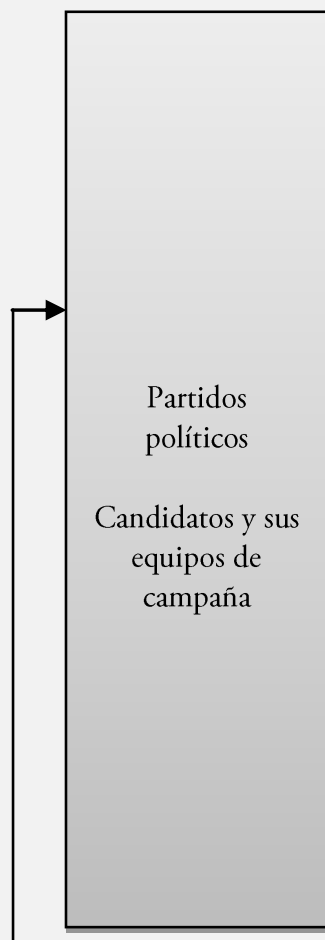

3) Canales de comunicación

\section{4) Efectos de las campańas}

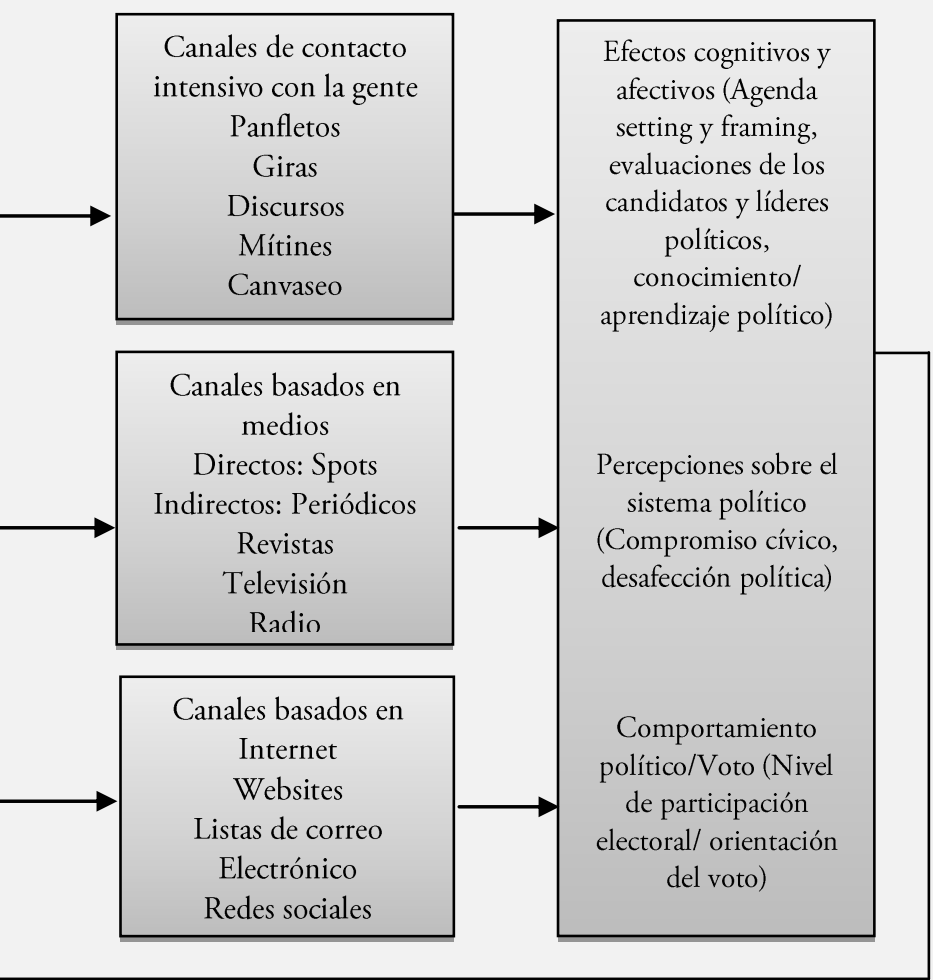

5) Retroalimentación

1) Ambiente contextual

Estructura del sistema de medios y del sistema de partidos

Fuente: Adaptado con base en Norris (2002, p. 128) y Vreese (2010, p. 120).

En México, al igual que en Estados Unidos, los efectos de las campañas electorales constituye el área que más ha sido investigada. Los politólogos y especialistas en comunicación de masas han dedicado la mayor parte de sus esfuerzos a evaluar el impacto de distintos tipos de fuentes de información durante las campañas en el conocimiento político, las actitudes políticas y el comportamiento 
político de los mexicanos. El objetivo de este artículo es revisar los hallazgos de tales estudios, así como señalar las áreas que, en comparación con otros contextos, han sido poco exploradas en relación con los efectos de las campańas mexicanas. No es la intención de este trabajo llevar a cabo una revisión exhaustiva de la literatura acerca del impacto de las comunicaciones políticas contemporáneas en América Latina, sino contribuir al debate académico del tema, a partir de una revisión sistemática de las investigaciones sobre el caso mexicano. Esperamos que el presente artículo invite a otros investigadores en comunicación política en la región a realizar revisiones críticas de los estudios sobre el impacto de diversos canales de comunicación política en la opinión pública, el comportamiento y la cultura política de los electores en sus respectivos países. Muchos de ellos son, al igual que México, terreno fértil para los efectos de los medios y las campañas debido a sus bajos o moderados niveles de identificación partidista, su alta concentración mediática y limitadas fuentes de información política disponibles para los ciudadanos.

\section{Las campañas electorales mexicanas y su modernización}

El 2 de julio de 2000, Vicente Fox, el candidato presidencial del Partido Acción Nacional (PAN), logró una dramática victoria en las elecciones presidenciales mexicanas que puso fin a uno de los regímenes autoritarios de mayor duración en el mundo. Después de siete décadas en el poder, el Partido Revolucionario Institucional (PRI) fue finalmente derrotado. Diversos factores de largo y mediano plazo explican su derrota, incluyendo cambios profundos en la economía política del país, en las instituciones electorales, así como en la estructura y el comportamiento del electorado mexicano, mismos que tuvieron lugar durante las últimas décadas del siglo XX. Sin embargo, el resultado de la elección no puede ser explicado sin tomar en consideración otros factores de corto plazo, relacionados con una serie de cambios en las tácticas y estrategias de campaña de los partidos de oposición (DOMÍNGUEZ, 2004). Como bien señala Klesner, el prolongado declive electoral del PRI refleja las debilidades de los partidos de oposición, incluyendo sus limitadas bases sociales de apoyo y "estrategias de campaña que aparentemente no pretendían ir más allá de esas bases” (KLESNER, 2005, p. 103). Seis años más tarde, el candidato presidencial del PAN, Felipe Calderón, conservaría la Presidencia de la República en una elección muy reñida en contra de Andrés Manuel López Obrador, candidato de una coalición de partidos de izquierda encabezada por el Partido de la Revolución Democrática (PRD). Una vez más, las tácticas y estrategias de campaña tuvieron un papel decisivo en la definición del resultado de la elección (DOMÍNGUEZ, 2009). 
Durante las últimas dos décadas, los principales partidos mexicanos han modernizado sus campañas electorales presidenciales de manera significativa, como resultado de cambios a gran escala en el sistema de partidos (KLESNER, 2005), en el sistema de medios de comunicación (LAWSON, 2002; HUGHES, 2006) y en el sistema de financiamiento político (BECERRA et al., 2000). Lo que antes era esencialmente una gira de varios meses por los estados de la república (LANGSTON y BENTON, 2009; LOMNITZ et al., 2010) ha dado paso a un estilo moderno, pero híbrido, de hacer campaña; éste combina prácticas tradicionales en las que se procura el contacto directo con los votantes (particularmente tácticas de movilización electoral sustentadas en redes de intercambio clientelar, people-intensive campaigning)con tácticas y estrategias modernas de campaña, basadas a su vez en el uso intensivo de consultores, sofisticadas técnicas de marketing político y de investigación de opinión pública, nuevas tecnologías de la información y la comunicación y, sobre todo, medios de comunicación de masas (media-intensive campaigning) ${ }^{2}$.

De manera similar a las tendencias de modernización de las campañas observadas en muchas democracias avanzadas y emergentes alrededor del mundo ${ }^{3}$, los medios de comunicación, en particular la televisión, se han convertido en el centro de una serie de esfuerzos y comunicaciones de campaña realizadas por los candidatos presidenciales de los tres grandes partidos mexicanos (WALLIS, 2001; LANGSTON y BENTON, 2009).

Aunque la eficacia de las tácticas y estrategias de campaña basadas en el uso intensivo de los medios es a menudo un tema de debate entre los estudiosos y comentaristas, numerosas investigaciones demuestran que (junto a las redes sociales y a otras organizaciones secundarias) los medios desempeñan un papel cada vez más importante como intermediarios electorales entre partidos y votantes en México (LAWSON, 1999; 2004a, 2004b; MORENO, 1999, 2002 y 2004; MORENO, 2009a, 2009b; POIRÉ, 1999; LAWSON y MCCANN, 2005; BELTRÁN, 2007;

\footnotetext{
${ }^{2}$ Un tema de particular interés en la literatura sobre el cambio en las campañas es el de la 'hibridación' de las prácticas de campaña, entendido como la "complementación específica en un país, de las prácticas tradicionales de campaña con características seleccionadas del estilo posmoderno, orientado a los medios" (PLASSER y PLASSER, 2002b, p. 374). La hibridación ocurre porque partidos y candidatos alrededor del mundo a menudo seleccionan aquellas innovaciones de campaña que son adecuadas para su contexto mediático y electoral específico, adaptándolas al mismo, con lo que suplementan, más que remplazan, las formas tradicionales de hacer campaña (para estudios de caso sobre la hibridación de las campañas en América Latina, véase De La Torre y Conaghan (2009).

3 Véase Bowler y Farrell (1992), Butler y Ranney (1992), Swanson y Mancini (1996), Gunther y Mughan (2000), Plasser y Plasser (2002a) y Schafferer (2006).
} 
VALENZUELA y MCCOMBS, 2007; FLORES-MACÍAS, 2009; GREENE, 2009; VALDIVIA y BELTRÁN, 2009).

\section{Los efectos de las comunicaciones de campaña en México}

El interés por los efectos de las comunicaciones políticas en las actitudes y el comportamiento político ha sido una constante en la ciencia política y los estudios en comunicación contemporáneos. Sin embargo, las investigaciones pioneras sobre el comportamiento político (LAZARSFELD et al., 1944; BERELSON et al., 1954) que llevaron al paradigma de los efectos minimos limitaron fuertemente el interés académico por los efectos de las campañas, de los medios, y de otros factores de corto plazo que influyen sobre las actitudes políticas y el comportamiento electoral en las décadas subsecuentes ${ }^{4}$. Así, pese a los cambios significativos en las tácticas y estrategias de campaña ocurridos durante la segunda mitad del siglo XX en los Estados Unidos, el paradigma de los efectos mínimos se mantuvo como dominante durante la mayor parte de ese periodo 5 . No obstante, durante los años ochenta del siglo pasado surgió un renovado interés por las campañas electorales, así como su impacto en la opinión pública y el comportamiento político, debido a diversos factores, entre los cuales se destacan los siguientes:

1. La percepción por parte de la comunidad académica de procesos de desalineamiento social y partidista, así como la mayor volatilidad electoral, lo cual llevó eventualmente a cuestionar las teorías que hacían énfasis en los aspectos de largo plazo que influyen sobre el voto, tales como la identificación partidista, y a reconsiderar los efectos de las variables de corto plazo, incluyendo las modernas comunicaciones de campaña en el comportamiento electoral.

2. La aparición de nuevos desarrollos teóricos sobre los efectos de los medios masivos en la década de los setenta, los cuales propusieron modelos alternativos de efectos mediáticos significativos, capaces de superar el proceso de 'percepción selectiva', y de producir cambios significativos en las cogniciones y el conocimiento político de las personas, tales como 'la espiral del silencio' (NOELLE-NEUMANN, 1973 y 1974), el 'establecimiento (o definición) de la agenda' y los efectos de priming y framing (MCCOMBS y

\footnotetext{
${ }^{4}$ Véase, para una revisión del modelo de los efectos limitados de los medios, Klapper (1960).

${ }^{5}$ Cabe señalar que los estudios pioneros sobre la influencia de las campañas en Estados Unidos se llevaron a cabo antes de la aparición de modernas y sofisticadas tácticas y estrategias de campaña, basadas en la medición científica de la opinión pública y orientadas al uso intensivo de medios masivos (KRAUS y DAVIS, 1976).
} 
SHAW, 1972; IYENGAR y KINDER, 1987; IYENGAR, 1991) ${ }^{6}$ La aparición de estos desarrollos teóricos fue en gran medida posible gracias a los avances metodológicos en la investigación sobre comunicación política ${ }^{7}$. El uso de sofisticados diseños y técnicas de investigación que combinan análisis de contenido con estudios de encuestas transversales y longitudinales, métodos cualitativos y experimentales, ha permitido capturar una amplia variedad de efectos de la comunicación política contemporánea.

Como resultado, un creciente número de estudios se han centrado en la influencia de los mensajes políticos provenientes de los medios y de las modernas comunicaciones de campaña en la opinión pública y el comportamiento político (POPKIN, 1991; ZALLER, 1992 y 1996; ANSOLABEHERE y IYENGAR, 1995; MUTZ, 1998). En este sentido, Vreese (2010) ofrece una tipología de efectos de los medios y de las campañas; así, los divide entre cognitivos y afectivos, aquellos relacionados con las percepciones de las personas sobre el sistema político y, por último, los que actúan sobre el comportamiento político (véase la Tabla 1). Esta tipología será la que utilizaremos en nuestra revisión de los efectos de las campañas en el caso mexicano.

Tabla 1 - Una tipología de los efectos de los medios y las campañas

\begin{tabular}{c|c|c|c}
\hline & $\begin{array}{c}\text { Efectos cognitivos y } \\
\text { afectivos }\end{array}$ & $\begin{array}{c}\text { Percepciones del } \\
\text { sistema político }\end{array}$ & $\begin{array}{c}\text { Comportamiento } \\
\text { político }\end{array}$ \\
\hline Agenda-setting y framing & $\times$ & & \\
\hline Evaluaciones de los líderes políticos & $\times$ & & \\
\hline Aprendizaje/ Conocimiento político & $\times$ & & \\
\hline Desafección política & & $\times$ & \\
\hline Eficacia política & & $\times$ & $\times$ \\
\hline Involucramiento político & & & $\times$ \\
\hline Participación política & & & \\
\hline Orientación del voto & & & \\
\hline
\end{tabular}

Fuente: Adaptado de Vreese (2010, p. 120).

\section{Canales de comunicación durante las campañas}

Las campañas electorales implican una serie de mensajes dirigidos a grupos determinados de electores; para ello se emplean tres canales principales de comunicación política: a) los canales tradicionales basados en el contacto directo con las personas (people-intensive channels), b) los modernos medios de comunicación de

\footnotetext{
${ }^{6}$ Véase, para una revisión sobre el tema, Bryant y Zillmann (1994), Norris et al. (1999) y Perse (2001).

${ }^{7}$ Véase Graber (2004).
} 
masas (media-intensive channels) y c) las nuevas tecnologías de la información y la comunicación, especialmente el Internet (Internet channels) (NORRIS, 2005) (véase la Gráfica 1). Este trabajo se concentra, principalmente, en los canales relacionados con el uso de medios de comunicación electrónicos como la televisión.

Los partidos y candidatos tienen dos vías de acceso a los medios, una de ellas es a través de los canales directos de comunicación (también llamados controlados), en los que pueden controlar el contenido de sus mensajes (ejemplo: spots). La otra forma es por medio de los llamados canales indirectos (o mediados), proporcionados por los medios de comunicación al realizar la cobertura noticiosa durante las campañas. Examinaremos a continuación los efectos de ambos tipos de canales de comunicación política en el contexto mexicano, de acuerdo con el esquema propuesto en la Tabla 1.

\section{Canales indirectos de comunicación: cobertura informativa}

\section{Efectos cognitivos y afectivos}

\section{Conocimiento politico}

Influyentes estudios comparados muestran que la exposición a mensajes de campaña puede incrementar los niveles de conocimiento político de los ciudadanos, incluso cuando se agregan controles sociodemográficos como la educación o el nivel socioeconómico (NORRIS, 2000). En el caso mexicano, algunos estudios (MORENO, 2003 y 2009a) han mostrado que la exposición a la información de las campañas puede generar también aumentos significativos en el interés, el aprendizaje y el involucramiento político de los electores. Por ejemplo, el análisis de Moreno (2002) sobre las campañas de los tres principales partidos en las elecciones para gobernador de 1999, en el Estado de México, demuestra que los votantes mexicanos obtienen y procesan la información y los mensajes políticos provenientes de las campañas de forma bastante comprensible (e incluso predecible), de acuerdo con las señales enviadas por los partidos políticos (partisan cues) y según sus predisposiciones políticas y niveles de atención a las campañas. El análisis sugiere que los electores percibieron los atributos de las imágenes de los candidatos que contendieron durante esa elección de manera muy similar a la de los candidatos que compitieron, en otras elecciones, con la etiqueta del mismo partido. Sin embargo, el autor señala que no es claro si esto se debe a que las señales partidistas se desarrollaron previamente en la mente de los votantes, o bien, porque los partidos siguen estrategias de comunicación política similares en distintas elecciones, o incluso, debido a ambos. 


\section{Establecimiento de la agenda (agenda-setting)}

Puesta en términos simples, la hipótesis del establecimiento de la agenda sostiene que hay una transferencia de prominencia temática de los medios hacia el público; en otras palabras, la "información sobre asuntos políticos, candidatos y otros temas que presentan las noticias se vuelve la información prioritaria dentro de la opinión pública sobre esos mismos asuntos, candidatos y temas" (KIOUSIS, 2003, p. 121). La investigación sobre agenda-setting es una de las más prolíficas en el campo de la comunicación política, particularmente en lo referente a los efectos cognitivos de los medios en la opinión pública ${ }^{8}$. La hipótesis del establecimiento de la agenda tuvo sus inicios a principios de los años setenta, con el estudio pionero de Maxwell McCombs y Donald Shaw (1972), realizado durante la elección presidencial estadounidense de 1968. De acuerdo con un análisis de contenido de los temas que privilegiaban los medios y de los datos de encuestas de opinión sobre los asuntos que privilegiaban las audiencias en esa elección, los autores encontraron un alto grado de correlación estadística entre ambas agendas temáticas, proporcionando así evidencia empírica sobre la existencia de una relación directa entre el contenido de la agenda de los medios y la subsecuente percepción, por parte del público, sobre cuáles eran los temas más importantes. A partir de entonces se han realizado más de 400 estudios empíricos alrededor del mundo que confirman, en mayor o menor medida, el efecto de agenda-setting de los medios (MCCOMBS, 2004). Cabe señalar que las investigaciones que siguen la tradición del establecimiento de la agenda acerca del impacto de los medios en las cogniciones y las actitudes de los mexicanos han sido (y son aún) escasas. Luego de revisar las investigaciones de los efectos de los medios en el caso mexicano, Issa Luna Pla (2003, p. 13) concluyó que hasta ese momento no habían "registros en México de los estudios basados específicamente en la teoría del agenda-setting, y son pocos los que mínimamente citan a sus teóricos fundamentales". Los primeros análisis desde esta perspectiva teórica se han enfocado en las consecuencias del proceso del establecimiento de la agenda en el comportamiento político (VALENZUELA y MCCOMBS, 2007).

\section{Comportamiento Politico}

Aunque la mayor parte de la investigación sobre el establecimiento de la agenda se ha concentrado en los efectos cognitivos y actitudinales del contenido de los medios, un creciente número de estudios realizados durante las últimas dos décadas ha explorado también las consecuencias del proceso de definición de la

\footnotetext{
${ }^{8}$ Véase, para una revisión, Dearing y Rogers (1996) y McCombs (2004).
} 
agenda en el comportamiento político. La idea que orienta tales análisis es que los cambios en la prominencia otorgada por los medios a determinados objetos sociales, y a determinados atributos sobre esos objetos, terminan por afectar el comportamiento de las personas ${ }^{9}$. En el caso mexicano, diversos estudios han analizado el impacto de la exposición a los medios informativos en las opiniones y el comportamiento electoral utilizando de manera explícita o implícita el marco teórico del establecimiento de la agenda (LAWSON, 1999, 2002 y 2004b; LAWSON y McCANN, 2005; VALENZUELA y MCCOMBS, 2007).

Los hallazgos de estas investigaciones muestran que, en contraste con el modelo de los 'efectos limitados' acerca de la influencia de los medios de comunicación en las campañas electorales, los efectos de los medios sobre las actitudes políticas y el comportamiento electoral de los mexicanos en las elecciones de 1997, 2000 y 2006 fueron significativos y sustanciales debido a las características del contexto político e informativo mexicano, relativas a las limitadas fuentes de información política y a la debilidad de las lealtades partidistas entre el electorado. Por ejemplo, Lawson (2002) muestra que la exposición de los electores a las noticias en Televisa disminuyó la probabilidad de votar por el PRI en la elección intermedia de 1997. En una investigación posterior, Lawson y McCann (2005) hallaron que la exposición a la cobertura de noticias de Televisa en la elección presidencial de 2000 tuvo, por el contrario, un efecto mucho más moderado en las actitudes y las preferencias de los votantes. Sin embargo, durante esa misma elección, la cobertura noticiosa de las campañas que realizó TVAzteca dañó significativamente la imagen del candidato del PRI, Francisco Labastida, frente a sus rivales (particularmente frente a Vicente Fox $)^{10}$. De acuerdo con el estudio, ver las noticias de TVAzteca se asoció con aumentos significativos en la probabilidad de votar por Fox y con descensos importantes en la probabilidad de apoyar a Labastida ${ }^{11}$.

Otros estudios sustentados en datos agregados de encuestas y de medición de audiencias (gross rating points) también confirman la existencia de efectos significativos de la cobertura mediática de las campañas y la publicidad política sobre

\footnotetext{
${ }^{9}$ Véanse, para una revisión, Kiousis (2003), McCombs (2004) y Valenzuela y McCombs (2007).

${ }^{10}$ Pese a que inicialmente la imagen de Cuauhtémoc Cárdenas fue también afectada negativamente por el tono desfavorable de la cobertura de Televisa sobre su campańa, eventualmente su imagen se recuperó una vez que la cadena mejoró de forma significativa el tono de la cobertura en la segunda mitad de la elección.

${ }^{11}$ En un estudio previo, Lawson (2004b) estimó que la exposición a noticias de TVAzteca produjo un efecto neto de 7.6 por ciento a favor de Fox, una proporción de votos ligeramente mayor al margen de victoria real de Fox sobre Francisco Labastida.
} 
las preferencias de los votantes en el año 2000. El análisis de Beltrán (2007) muestra que el diferencial en la difusión de mensajes de campaña acumulados tuvo un efecto desfavorable para el candidato del PRI. No obstante, en contraste con los hallazgos del estudio de Lawson y McCann (2005), su análisis no encontró evidencia alguna de un efecto diferenciado de la cobertura de las dos cadenas principales de televisión sobre el comportamiento electoral ${ }^{12}$.

Más recientemente, Valenzuela y McCombs (2007) analizaron la influencia de la cobertura de noticias sobre las campañas de los candidatos presidenciales en las decisiones de los votantes en la elección de 2006, con la teoría del establecimiento de la agenda, explícitamente, como marco de análisis ${ }^{13}$. Los autores usaron datos agregados de encuestas de opinión pública y análisis de contenido de la cobertura de la campaña en las dos cadenas nacionales de televisión. El estudio encontró que tanto Televisa como TVAzteca dieron mayor cobertura al panista Felipe Calderón que a sus rivales: Andrés Manuel López Obrador, del PRD, y Roberto Madrazo, del PRI. Además, mientras que el tono de la cobertura noticiosa fue claramente favorable para Calderón y para Madrazo, fue marcadamente desfavorable para el candidato de la izquierda.

Valenzuela y McCombs (2007) utilizaron análisis de regresión para medir los efectos del contenido de las noticias en las preferencias de los votantes. Sus resultados indican que aproximadamente la mitad de la variación en las decisiones de voto podría explicarse por la cobertura mediática de las campañas. En resumen, mientras más favorable fue la cobertura informativa dela campaña de un candidato, mayor fue el porcentaje de apoyo hacia éste por parte delos electores ${ }^{14}$. Al igual que el estudio de

${ }^{12} \mathrm{Su}$ trabajo sugiere que los efectos de la exposición a la cobertura de TVAzteca encontrados por Lawson y McCann (2005) se deben a una sobreestimación respecto de la exposición a las noticias de TVAzteca por parte de los votantes, de acuerdo con el uso de datos de encuesta.

${ }^{13}$ Los autores replicaron un estudio previo realizado por Son y Weaver (2006), el cual examinó los efectos del proceso del establecimiento de la agenda en el comportamiento electoral, en la elección presidencial de 2000 en EE.UU., y que encontró evidencia de que el tono afectivo de los atributos altamente sobresalientes en la opinión pública de los principales candidatos presidenciales influyó en el apoyo hacia los candidatos en las encuestas durante la campaña electoral.

${ }_{14}$ No obstante, debido a que su diseño de investigación asume que las preferencias de los electores pueden predecirse enteramente de acuerdo conla información que ellos reciben sobre los candidatos, Valenzuela y McCombs (2007) advierten que el modelo puede sobreestimar los efectos de los medios y esconder la influencia de otros factores tales como la economía, la popularidad del partido en el gobierno, etcétera. Por lo tanto, futuras investigaciones deben incluir condiciones del mundo real (real-world conditions) como variables de control, así como explorar la influencia de otros tipos de canales de comunicación política sobre los cambios en la opinión pública. 
Beltrán (2007), su investigación concluye que los efectos significativos de los medios ocurrieron acumulativamente, durante periodos prolongados de tiempo y no de manera inmediata. Los autores también señalan que un análisis más completo del impacto de las campañas requeriría analizar los efectos de la cobertura de noticias y de la publicidad política, ya que ésta pudo haber desempeñado un papel relevante al reforzar algunos atributos de los candidatos durante la campańa.

\section{Canales directos de comunicación: publicidad politica}

La investigación comparada sobre publicidad política da cuenta del papel central que la televisión juega en las estrategias modernas de campaña alrededor del mundo (KAID y HOLTZ-BACHA, 1995 y 2006). El caso mexicano no es la excepción. De 1997 a 2006, los candidatos presidenciales de los principales partidos invirtieron la mayor parte de sus presupuestos de campaña en spots pagados en radio y televisión (LOZANO, 2006; LAWSON, 2008). Sin embargo, en su revisión acerca de la investigación sobre publicidad política en México, Lozano (2006, p. 263) concluyó que, "a pesar de su creciente uso a partir de la elección de 1994, la publicidad política es uno de los tópicos menos estudiados en la literatura académica sobre política y medios en México".

Este vacío en la literatura se ha ido llenando gradualmente por una serie de estudios como el de Beltrán (2007), que trata los efectos combinados de la exposición a noticias y a spots en la elección presidencial de 2000. El autor argumenta que los efectos de la cobertura informativa y de la publicidad política no deben ser analizados en forma aislada, debido a que ambos canales de comunicación política actúan conjuntamente a través de un proceso acumulativo y limitándose mutuamente durante las campañas. De acuerdo con su análisis, aunque la publicidad política tuvo un impacto significativo en las preferencias de los votantes, la exposición a las noticias tuvo un impacto aún mayor que el de los spots.

El estudio de los canales directos de comunicación durante las campañas mexicanas también se ha aproximado a los efectos de la publicidad negativa. Un ejemplo son los análisis de Moreno (2003), quien ofrece evidencia del impacto diferenciado de los canales directos e indirectos de comunicación política en esa elección, particularmente sobre la probabilidad de votar de los electores. Mientras la exposición a noticias fue la variable de mayor importancia para explicar la probabilidad de votar, superando incluso el impacto de la intensidad del partidismo 
de los electores ${ }^{15}$, la percepción de la negatividad en las campañas actuó en detrimento de la participación electoral ${ }^{16}$. Sin embargo, la negatividad no afectó a todos los electores de igual manera: los spots negativos de la campaña de Vicente Fox desmovilizaron a algunos de los partidistas del PRI, al tiempo que la publicidad negativa del candidato priista no logró dañar la imagen de su rival. Por el contrario, ésta tuvo un efecto desmovilizador sobre sus propios partidistas hacia el final de la contienda, muchos de los cuales decidieron abandonar a Labastida en su intento por ganar la presidencia, ya sea votando por Fox o simplemente al abstenerse de votar (MORENO, 2004) $)^{17}$.

Empero, investigaciones subsecuentes sostienen que los efectos de las campañas negativas tanto en la participación electoral como en la orientación del voto fueron más limitados durante la elección presidencial de 2006. El análisis de Guerrero y Arellano (2012) indica que de la totalidad de spots negativos transmitidos por los dos candidatos punteros y sus partidos en esa elección, sólo los spots de López Obrador tuvieron un impacto significativo y positivo (aunque moderado) en la participación electoral. Cabe señalar que el estudio no encontró evidencia de que este efecto de movilización haya favorecido a alguno de los candidatos.

Otros estudios se han enfocado en los efectos que tienen en el voto los canales de comunicación de campaña basados en medios (o de aire), frente al impacto de los canales que se apoyan en el contacto directo con los electores mediante redes (o de tierra). La investigación de Valdivia y Beltrán (2009) referente al impacto de ambos canales de comunicación en la elección de 2006 encontró que las redes de comunicación vecinales tuvieron un impacto significativo en las preferencias de los electores por López Obrador. Por el contrario, el estudio no encontró evidencia alguna de que la comunicación dentro de las redes sociales haya influido de manera

\footnotetext{
${ }^{15}$ Su análisis muestra que la intensidad del partidismo de los electores explica una mayor propensión a votar el día de la elección para cualquier grado de exposición a medios. Sin embargo, la exposición a noticias media la relación entre partidismo y participación electoral de manera muy relevante en esa elección. Por ejemplo, "un elector independiente con grado medio o alto de exposición a medios es más probable de votar que un partidista duro o blando con bajos niveles de exposición a medios" (MORENO, 2003, p. 160).

${ }^{16}$ El coeficiente de percepción sobre publicidad negativa estuvo muy cerca de alcanzar significancia estadística al 0,05\%.

17 De acuerdo con Moreno, la brecha de negatividad, es decir, la percepción de que un determinado candidato ataca a sus rivales y que éstos (sus adversarios), por el contrario, no lo hacen, aumentó las posibilidades de cambiar de Labastida a Fox, cuando quien tenía la brecha era Labastida. Sin embargo, esto no influyo en las posibilidades de cambiar el sentido del apoyo de Fox a Labastida cuando el primero era quien tenía la brecha.
} 
directa en las preferencias por Calderón. El análisis también muestra que la publicidad política en medios resultó eficaz para la campaña del candidato del PAN; no obstante, ésta no parece haber beneficiado a la campaña del candidato del PRD. Los autores sugieren que la ineficacia de la campaña en medios del candidato perredista se puede deber al hecho de que sus mensajes no se enfocaron en un tema en particular y sólo se acumularon en la parte final de su campaña. Por el contrario, la campaña de Calderón fue más efectiva porque centró su estrategia en el uso de publicidad en medios masivos con dos o tres mensajes claros, todos relacionados con la economía, los cuales fueron comunicados de manera continua y consistente a lo largo de la campaña.

\section{Evaluaciones de los candidatos}

¿Cómo se pueden explicar los efectos significativos de las comunicaciones de campaña en el comportamiento electoral en México? ¿Mediante cuáles mecanismos influyen las comunicaciones de campaña en las decisiones electorales? ¿Los mensajes de campaña persuadieron, activaron o simplemente actuaron como un reforzamiento de las actitudes previas de los votantes? Diversos estudios sobre el caso mexicano muestran que las comunicaciones de campaña influyen de manera significativa en los estándares con los que los votantes evalúan a los gobiernos y a los candidatos a través del énfasis en temas particulares, tales como la economía (un proceso llamado priming).

De acuerdo con la teoría del voto económico retrospectivo, los electores tienden a premiar con su voto el buen desempeño económico de los gobiernos y a retirarles su apoyo cuando perciben que la economía anda mal (FIORINA, 1981) ${ }^{18}$. No obstante, análisis comparados recientes sobre el voto económico muestran una variación considerable en cuanto a la magnitud del impacto de la economía en las decisiones de los votantes, tanto en democracias establecidas como en desarrollo (DUCH y STEVENSON, 2008; HELLWIG, 2010). Estos trabajos muestran que la propensión que tienen los votantes de vincular la situación de la economía con sus decisiones de voto es susceptible de ser influida por diversos factores tanto estructurales como institucionales, así como por las acciones estratégicas de las elites políticas, las cuales "pueden hacer uso de sus poderes asimétricos de control de la información, de acceso a los medios de comunicación y de formulación de políticas

\footnotetext{
${ }^{18}$ Para la mayor parte de la literatura sobre voto económico, son los factores macroeconómicos, así como la percepción que los electores tienen del estado de la economía, y no los esfuerzos de campaña de partidos y candidatos, los principales responsables de su éxito (o fracaso) en elecciones presidenciales.
} 
públicas, para modular el grado en que una elección se decide por motivos económicos" (HELLWIG, 2010, p. 201).

En este sentido, Hellwig (2010) sostiene que la investigación comparada sobre la influencia de la economía en el comportamiento político debe analizar con mayor profundidad el papel de las acciones estratégicas de las elites políticas, ya que éstas usualmente envían mensajes políticos a los votantes diseñados con la finalidad de aumentar su rendimiento electoral o minimizar el de sus competidores. Un creciente número de estudios muestra que los mensajes de campaña pueden mediar entre el estado de la economía y las decisiones de los votantes (VAVRECK, 2009). Por ejemplo, Vavreck (2009) demuestra que los candidatos de partidos en el gobierno con una buena gestión de la economía y los candidatos de oposición en una mala situación económica tienen usualmente una ventaja importante en elecciones presidenciales.

Sin embargo, la autora advierte que no es suficiente tener la economía a favor para ganar una elección. La historia provee ejemplos recientes de candidatos que perdieron elecciones aun con el tema de la economía de su lado (por ejemplo, Al Gore en la elección presidencial estadounidense de 2000 y, en el caso mexicano, Francisco Labastida). Por lo tanto, para ganar, este tipo de candidatos tiene que explotar esa ventaja a través de mensajes de campaña que enfaticen el tema de la economía por encima de cualquier otro, y hacer suficientemente claro para los votantes el vínculo que existe entre la administración de su partido y una buena situación económica, o entre el partido de su adversario y una mala gestión de la economía. En el caso mexicano, distintos estudios muestran la importancia de la interacción entre las comunicaciones de campaña y las evaluaciones retrospectivas de los votantes (GREENE, 2009 y 2011; MORENO, 2009b; HART, 2013).

Moreno (2009b) sostiene que, a diferencia de anteriores contiendas presidenciales, el voto económico fue un aspecto particular de la elección presidencial de $2006^{19}$. Sin embargo, los razonamientos de tipo económico por parte de los electores no surgieron de manera espontánea, sino que fueron en buena medida activados por los mensajes de campaña durante la elección, particularmente por los

\footnotetext{
${ }^{19}$ Estudios previos sobre el voto económico en México muestran que las evaluaciones retrospectivas tuvieron cierta influencia en el comportamiento electoral durante la década de 1990 (MAGALONI, 1999; POIRÉ, 1999; BUENDÍA, 2004). Sin embargo, su impacto fue mucho más limitado respecto al que usualmente tienen en las democracias establecidas, ya que el PRI se mantuvo en el gobierno pese a las profundas crisis económicas de mediados los ochenta. La intención de voto a favor del PRI siguió siendo alta incluso entre aquellos electores que expresaron mayor insatisfacción con su rendimiento económico (DOMÍNGUEZ y MCCANN, 1996; BUENDÍA, 2004; MAGALONI, 2006).
} 
mensajes de la campaña del candidato del partido en el gobierno. Al comienzo de la elección presidencial de 2006, la mayoría de las encuestas mostraba una ventaja de López Obrador sobre Felipe Calderón de alrededor de diez puntos porcentuales, la cual se mantuvo hasta finales de marzo, a pesar de los esfuerzos de la campaña del panista. Incluso sus propias encuestas internas revelaron que la estrategia inicial de campaña, consistente en presentar a Calderón como un candidato con las 'manos limpias' y con 'pasión y valor por México', no estaba funcionando. Como resultado, Calderón realizó cambios significativos en su equipo y estrategia de campaña (BRUHN, 2009; TREJO DELARBRE, 2010). Según los resultados de encuestas y grupos de enfoque, su equipo reorientó los mensajes de la campaña hacia temas económicos, presentándolo como 'el presidente del empleo' y destacando la popularidad y el relativamente buen desempeño macroeconómico de la administración del presidente Fox. De manera paralela, el PAN lanzó una intensa campaña negativa contra López Obrador, acusándolo de ser un peligroso populista, intolerante e incompetente que, en caso de ganar la elección, llevaría al país a una crisis económica (FREIDENBERG y GONZÁLEZ, 2009).

Moreno plantea que, al enmarcar la contienda electoral como una decisión entre la estabilidad macroeconómica y la generación de empleos, por un lado, y el endeudamiento y la crisis económica, por el otro, las comunicaciones de la campaña panista "tuvieron como efecto la vinculación de los puntos de vista sobre la economía con las preferencias electorales” (MORENO, 2009b, p. 270) ${ }^{20}$. En resumen, los mensajes de la campaña del PAN activaron un razonamiento de tipo económico en los electores que iba en este sentido: si se consideraba que la economía marchaba bien, entonces el apoyo a Calderón era mayor; por el contrario, si se pensaba que la economía iba mal, el apoyo era para López Obrador. Sin embargo, Moreno señala que la naturaleza del efecto de los mensajes de campaña fue de activación de las predisposiciones latentes de los votantes y no de persuasión (lo cual implica cambiar sus puntos de vista), ya que la campaña panista no logró convencer a aquellos que pensaban que la economía funcionaba mal de lo contrario, sino que apeló al voto económico de los que ya consideraban que la economía iba bien. Esto, aun así, no es irrelevante, ya que una buena parte de estos electores tenían la intención de votar por López Obrador al inicio de la elección.

\footnotetext{
${ }^{20}$ Un mes después del cambio en la estrategia de comunicación del candidato panista, la mayoría de las encuestas de opinión pública mostraron que Calderón estaba alcanzando a López Obrador, y que la diferencia entre ambos era de menos de cinco por ciento, la cual siguió estrechándose hasta que, a principios de mayo, el candidato del PAN apareció como puntero por primera vez en la contienda, misma que terminaría ganando por un margen de menos de un punto porcentual.
} 
En una línea de argumentación similar, el estudio de Heart (2013) sobre la influencia de las comunicaciones de campaña en la activación del voto económico durante las elecciones presidenciales de 2000 y 2006, también encontró evidencia de que la publicidad política centrada en la economía condicionó fuertemente las evaluaciones económicas retrospectivas de los electores en 2006. Empleando datos del Estudio Panel México 2000 y 2006 y análisis de contenido de la publicidad política en ambas elecciones, Heart demuestra que cuando los mensajes de campaña centrados en la economía constituyen sólo un pequeño porcentaje de la publicidad política a la cual los electores son expuestos, sus opiniones sobre la economía no parecen tener impacto alguno en sus preferencias electorales, tal y como sucedió en la elección presidencial de 2000. Por el contrario, el aumento en la exposición de los electores a spots enfocados en temas económicos aumenta significativamente el impacto de las evaluaciones retrospectivas en sus decisiones de voto. En el caso de la elección de 2006, mientras el flujo de publicidad focalizada en temas económicos se incrementó durante la campaña, las evaluaciones sobre la economía se volvieron cada vez más un predictor del apoyo electoral para Calderón.

Por su parte, Greene (2009) sostiene que la campaña de Calderón posicionó de forma exitosa el tema de la crisis y, aunque el enfatizar este tema en particular no necesariamente persuadió a más votantes de temer una victoria de López Obrador, sí hizo que aquellos que ya estaban preocupados acerca de la crisis pusieran más peso en ese factor al decidir cómo votar. En otras palabras, un votante que estaba de acuerdo con que López Obrador era un riesgo pero aún planeaba votar por este candidato, debido a sus actitudes respecto a otros factores al inicio de la elección, pudo haber sido predispuesto (primed) para votar por Calderón al final.

A diferencia de Moreno y Heart, Greene (2009) sostiene que los efectos de la campaña de 2006 van más allá de la mera activación de predisposiciones latentes en el electorado. Su análisis muestra que los mensajes de campaña de Calderón también persuadieron a los votantes de que el panista era más capaz en diversas áreas de política pública, tales como la seguridad pública, el combate a la corrupción y, sobre todo, la gestión de la economía. Sólo un tema de políticas fue favorable a López Obrador: la reducción de la pobreza. A su vez, tales percepciones sobre las competencias de los candidatos acerca de esos temas influenciaron las decisiones de voto de manera significativa. En otras palabras, la exposición a las comunicaciones de campaña afectó notablemente las evaluaciones de los electores sobre las capacidades de los candidatos y predispuso a los votantes a darle más peso a la competencia en la gestión de la economía al momento de decidir por quien votar. 


\section{Conclusiones}

El análisis de los efectos de los medios es un área de rápido desarrollo en la investigación académica sobre comunicación política en México, cuyos hallazgos son de gran relevancia para la investigación en comunicación política comparada. Aunque difieren en cuanto a la metodología y las variables independientes utilizadas, así como en su concepción de la naturaleza del efecto causado (ya sea de reforzamiento, activación y/o persuasión), la mayor parte de los trabajos revisados en este artículo encuentran efectos significativos y considerables (quizás hasta decisivos) de las comunicaciones de campaña en las actitudes y el comportamiento político de los mexicanos.

En general, los hallazgos de estas investigaciones demuestran que el modelo de los efectos limitados, que influyó fuertemente la investigación sobre el impacto de los medios y de las campañas en el comportamiento político en democracias establecidas, puede no ser del todo adecuado para explicar el impacto de los medios y las comunicaciones de campaña en nuevas democracias con bajos niveles de identificación partidista, alta concentración mediática y limitadas fuentes de información política. Sin embargo, al igual que en el caso estadounidense, la evidencia sobre el tema es aún contradictoria: mientras que unas investigaciones muestran efectos significativos y substanciales de las comunicaciones de campaña en las actitudes y el comportamiento político, otros análisis difieren en cuanto a la existencia o la magnitud de tales efectos. Por otro lado, algunas áreas de la investigación sobre efectos de las comunicaciones políticas aún han sido poco desarrolladas, si no es que prácticamente no estudiadas, tales como el efecto de las comunicaciones de campaña sobre dimensiones relevantes del compromiso cívico de los mexicanos, incluyendo sus niveles de confianza política, de eficacia política y diversos aspectos de la participación ciudadana. Incluso, podría decirse que son inexistentes los estudios que pongan a prueba las teorías del 'video malestar' y de la 'movilización política', las cuales sostienen el impacto negativo y positivo de las comunicaciones políticas en el compromiso y la participación política de los ciudadanos $^{21}$.

\footnotetext{
${ }^{21}$ Dentro de las diferentes teorías de los efectos de los medios y las campañas se encuentran las teorías del 'malestar mediático' (media malaise) o 'video malestar' (video malaise) y las teorías de la movilización política. Las teorías del videomalestar sostienen que la exposición a las comunicaciones políticas modernas, sobre todo aquellas que se caracterizan por la personalización y la negatividad pueden generar mayor desafección política entre los ciudadanos. Por el contrario, las teorías de la movilización sostienen que existe una asociación positiva entre la exposición a los mensajes políticos y el compromiso cívico de las personas.
} 
Oniel Francisco Díaz Jiménez é Professor e Pesquisador do Departamento de Procesos Sociais da Universidade Autônoma Metropolitana, Unidade Lerma.E-mail: o.diaz@correo.ler.uam.mx

Igor Vivero Ávila é Professor e Pesquisador da Faculdade de Ciências Políticas e Sociais da Universidade Autônoma do Estado do México. E-mail: ivivero@yahoo.com

\section{Referencias}

ANSOLABEHERE, Stephen; IYENGAR, Shanto. Going Negative: How Attack Ads Shrink and Polarize the Electorate. New York: Free Press, 1995.

BECERRA, Ricardo; SALAZAR, Pedro; WOLDENBERG, José. La Mecánica del Cambio Politico en México: Elecciones, Partidos y Reformas. México City: Cal y Arena, 2000.

BELTRÁN, Ulises. The Combined Effect of Advertising and News Coverage in the Mexican Presidential Campaign of 2000. Political Communication, v. 24, n. 1, p. 37-63, 2007.

BERELSON, Bernard; LAZARSFELD, Paul F.; MCPHEE, William N. Voting: A Study of Opinion Formation in a Presidential Campaign. Chicago: University of Chicago Press, 1954.

BOWLER, Shaun; FARRELL, David M. (Orgs.). Electoral Strategies and Political Marketing. Basingstoke: Macmillan, 1992.

BRUHN, Kathleen. López Obrador, Calderón, and the 2006 Presidential Campaign. In: DOMÍNGUEZ, Jorge I.; LAWSON, Chappell; MORENO, Alejandro (Orgs.). Consolidating Mexico's Democracy: The 2006 Presidential Campaign in Comparative Perspective. Baltimore: Johns Hopkins University Press, 2009. p. 169-188.

BRYANT, Jennings; ZILLMANN, Dolf. Media Effects: Advances in Theory and Research. Hillsdale: L. Erlbaum Associates, 1994.

BUENDÍA, Jorge. The Changing Mexican Voter, 1991-2000. In: MIDDLEBROOK, Kevin J. (Org.). Dilemmas of Political Change in Mexico. San Diego: Institute of Latin American Studies, University of London/Center for U.S. - Mexican Studies, University of California San Diego, 2004. p. 108-129.

BUTLER, David; RANNEY, Austin (Orgs.). Electioneering: A Comparative Study of Continuity and Change. Oxford: Clarendon Press, 1992.

DE LA TORRE, Carlos; CONAGHAN, Catherine. The Hybrid Campaign. The International Journal of Press/Politics, v. 14, n. 3, jul. p. 335-352, 2009.

DEARING, James W.; ROGERS, Everett M. Agenda-setting. Thousand Oaks: Sage, 1996.

DOMÍNGUEZ, Jorge I. Conclusion: Why and How Did Mexico's 2000 Presidential Election Campaign Matter? In: DOMÍNGUEZ, Jorge I.; LAWSON, Chappell H. (Orgs.). Mexico's Pivotal Democratic Election: Candidates, Voters, and the Presidential Campaign of 2000. Stanford: Stanford University Press, 2004. p. 321-344. 
Conclusion: The Choices of Voters during the 2006 Presidential Election in Mexico. In: DOMÍNGUEZ, Jorge I.; LAWSON, Chappell H.; MORENO, Alejandro (Orgs.). Consolidating Mexico's Democracy: The 2006 Presidential Campaign in Comparative Perspective. Baltimore: Johns Hopkins University Press, 2009. p. 285-303.

DOMÍNGUEZ, Jorge I.; McCANN, James A. Democratizing Mexico: Public Opinion and Electoral Choices. Baltimore: Johns Hopkins University Press, 1996.

DUCH, Raymond M.; STEVENSON, Randolph T. The Economic Vote: How Political and Economic Institutions Condition Election Results. New York: Cambridge University Press, 2008.

FARRELL, David M. Campaign Strategies and Tactics. In: LEDUC, Lawrence; NIEMI, Richard G.; NORRIS, Pippa (Orgs.). Comparing Democracies: Elections and Voting in Global Perspective. Thousand Oaks: Sage, 1996. p. 160-183.

FIORINA, Morris P. Retrospective Voting in American National Elections. New Haven: Yale University Press, 1981.

FLORES-MACÍAS, Francisco. Electoral Volatility in 2006. In: DOMÍNGUEZ, Jorge I.; LAWSON, Chappell H.; MORENO, Alejandro (Orgs.). Consolidating Mexico's Democracy: The 2006 Presidential Campaign in Comparative Perspective. Baltimore: Johns Hopkins University Press, 2009. p. 191-208.

FREIDENBERG, Flavia; GONZÁLEZ, Luis. Estrategias partidistas, preferencias ciudadanas y anuncios televisivos: un análisis de la campaña electoral mexicana de 2006. Política y Gobierno, Ciudad del México, v. XVI, n. 2, jul-dez. p. 269-320, 2009.

GRABER, Doris A. Methodological Developments in Political Communication Research. In: KAID, Lynda Lee (Org.). Handbook of Political Communication Research. Mahwah: Lawrence Erlbaum Associates, 2004. p. 45-67.

GREENE, Kenneth F. Images and Issues in Mexico's 2006 Presidential Election. In: DOMÍNGUEZ, Jorge I.; LAWSON, Chappell H.; MORENO, Alejandro (Orgs.). Consolidating Mexico's Democracy: The 2006 Presidential Campaign in Comparative Perspective. Baltimore: Johns Hopkins University Press, 2009. p. 246-267.

. Campaign Persuasion and Nascent Partisanship in Mexico's New Democracy. American Journal of Political Science, East Lansing, v. 55, n. 2, p. 398-416, 2011.

GUERRERO, Manuel Alejandro; ARELLANO TOLEDO, Marco. Campañas negativas en 2006: ¿Cómo afectaron el voto? Ciudade del México: Universidad Iberoamericana, 2012.

GUNTHER, Richard; MUGHAN, Anthony (Orgs.). Democracy and the Media: A Comparative Perspective. Cambridge: Cambridge University Press, 2000.

HART, Austin. Can Candidates Activate or Deactivate the Economic Vote? Evidence from Two Mexican Elections. The Journal of Politics, West Nyack, v. 75, n. 4, p. 1051-1063, 2013.

HELLWIG, Timothy. Elections and the Economy. In: LEDUC, Lawrence; NIEMI, Richard G.; NORRIS, Pippa (Orgs.). Comparing Democracies 3: Elections and Voting in the $21^{\text {st }}$ Century. London: Sage, 2010. p. 184-201.

HUGHES, Sallie. Newsrooms in Conflict: Journalism and the Democratization of Mexico. Pittsburgh: University of Pittsburgh Press, 2006.

IYENGAR, Shanto. Is Anyone Responsible? How Television Frames Political Issues. Chicago: University of Chicago Press, 1991.

IYENGAR, Shanto; KINDER, Donald R. News that Matters: Television and American Opinion. Chicago: University of Chicago Press, 1987. 
KAID, Lynda Lee; HOLTZ-BACHA, Christina (Orgs.). Political Advertising in Western Democracies: Parties \& Candidates on Television. Thousand Oaks: Sage, 1995.

. The SAGE Handbook of Political Advertising. Thousand Oaks: Sage, 2006.

KIOUSIS, Spiro. Consecuencias cognoscitivas, actitudinales y conductuales del agenda-setting. In: McCOMBS, Maxwell; LUNA PLA, Issa (Orgs.). Agenda-setting de los medios de comunicación. Mexico: Universidad de Occidente, 2003. p. 121-137.

KLAPPER, Joseph T. The effects of mass communication. New York: Free Press, 1960.

KLESNER, Joseph L. Electoral Competition and the New Party System in Mexico. Latin American Politics \& Society, v. 47, n. 2, p. 103-142, 2005.

KRAUS, Sidney; DAVIS, Dennis K. The Effects of Mass Communication on Political Behavior. University Park: Pennsylvania State University Press, 1976.

LANGSTON, Joy; BENTON, Allyson. "A ras de suelo": Apariciones de candidatos y eventos en la campaña presidencial de México. Política y Gobierno, número especial, p. 135-176, 2009.

LAWSON, Chappell. Why Cárdenas Won: The 1997 Elections in Mexico City. In: DOMÍNGUEZ, Jorge I.; POIRÉ, Alejandro (Orgs.) Toward Mexico's Democratization: Parties, Campaigns, Elections, and Public Opinion. London: Routledge, 1999. p. 147-173.

Building the Fourth Estate: Democratization and the Rise of a Free Press in Mexico. Berkeley: University of California Press, 2002.

Mexico's Great Debates: The Televised Candidate Encounters of 2000 and Their Electoral Consequences. In: DOMÍNGUEZ, Jorge I.; LAWSON, Chappell (Orgs.). Mexico's Pivotal Democratic Election: Candidates, Voters, and the Presidential Campaign of 2000. Stanford, CA: Stanford University Press, 2004a. p. 211-240.

. Television Coverage, Vote Choice, and the 2000 Campaign. In: DOMÍNGUEZ, Jorge I.; LAWSON, Chappell (Orgs.). Mexico's Pivotal Democratic Election: Candidates, Voters, and the Presidential Campaign of 2000. Stanford: Stanford University Press, 2004b. p. 187-209.

Election Coverage in Mexico. In: STRÖMBÄCK, Jesper; KAID, Lynda Lee (Orgs.). The Handbook of Election News Coverage around the World. London: Routledge, 2008. p. 370-384.

LAWSON, Chappell; McCANN, James A. Television News, Mexico's 2000 Elections and Media Effects in Emerging Democracies. British Journal of Political Science, Colchester, v. 35, n. 1, p. 130, 2005.

LAZARSFELD, Paul Felix; BERELSON, Bernard; GAUDET, Hazel. The People's Choice: How the Voter Makes Up His Mind in a Presidential Campaign. New York: Duell, Sloan, and Pearce, 1944.

LOMNITZ, Larissa Adler de; SALAZAR ELENA, Rodrigo; ADLER, Ilya. Symbolism and Ritual in a One-Party Regime: Unveiling Mexico's Political Culture. Tucson: University of Arizona Press, 2010.

LOZANO, José Carlos. Political Advertising in Mexico. In: KAID, Lynda Lee; HOLTZ-BACHA, Christina (Orgs.). The SAGE Handbook of Political Advertising. London: Sage, 2006. p. 259-267.

LUNA PLA, Issa. Prólogo. In: McCOMBS, Maxwell y LUNA PLA, Issa (Orgs.). Agenda-setting de los medios de comunicación. México: Universidad de Occidente, 2003. p. 11-26.

MAGALONI, Beatriz. Is the PRI Fading? Economic Performance, Electoral Accountability, and Voting Behavior in the 1994 and 1997 Elections. In: DOMÍNGUEZ, Jorge I.; POIRÉ, Alejandro (Orgs.). Toward Mexico's Democratization: Parties, Campaigns, Elections, and Public Opinion. London: Routledge, 1999. p. 203-236. 
Voting for Autocracy: Hegemonic Party Survival and its Demise in Mexico. Cambridge: Cambridge University Press, 2006.

McCOMBS, Maxwell E. Setting the Agenda: The Mass Media and Public Opinion. Cambridge: Polity, 2004.

McCOMBS, Maxwell E.; SHAW, Donald L. The Agenda-Setting Function of Mass Media. The Public Opinion Quarterly, v. 36, n. 2, p. 176-187, 1972.

MORENO, Alejandro. Campaign Awareness and Voting in the 1997 Mexican Congressional Elections. In: DOMÍNGUEZ, Jorge I. y POIRÉ, Alejandro (Orgs.). Toward Mexico's Democratization: Parties, Campaigns, Elections, and Public Opinion. New York: Routledge, 1999. p. 114-146.

. Partisan Cues, Candidate Images, and Political Messages: How Mexican Voters Assimilate Campaign Information. Working Papers on Political Science. n. 3. Ciudad del México: Political Science Department, Instituto Tecnológico Autónomo de México, 2002.

El Votante Mexicano: Democracia, Actitudes Políticas y Conducta Electoral. Ciudad del México: Fondo de Cultura Económica, 2003.

. The Effects of Negative Campaigns on Mexican Voters. In: DOMÍNGUEZ, Jorge I.; LAWSON, Chappell (Orgs.). Mexico's Pivotal Democratic Election: Candidates, Voters, and the Presidential Campaign of 2000. Stanford: Stanford University Press, 2004. p. 243-268.

La Decisión Electoral: Votantes, Partidos y Democracia en México. Ciudad del México: Miguel Ángel Porrúa, 2009a.

. The Activation of Economic Voting in the 2006 Campaign. In: DOMÍNGUEZ, Jorge I.;

LAWSON, Chappell; MORENO, Alejandro (Orgs.). Consolidating Mexico's Democracy: The 2006

Presidential Campaign in Comparative Perspective. Baltimore: Johns Hopkins University Press, 2009b. p. 209-228.

MUTZ, Diana Carole. Impersonal Influence: How Perceptions of Mass Collectives Affect Political Attitudes. Cambridge: Cambridge University Press, 1998.

NOELLE-NEUMANN, Elisabeth. Return to the Concept of Powerfull Media. Studies of Broadcasting, v. 9, p. 66-112, 1973.

The Spiral of Silence: A Theory of Public Opinion. Journal of Communication, v. 24, n. 2, p. 43-51, 1974.

NORRIS, Pippa. A Virtuous Circle: Political Communications in Postindustrial Societies. Cambridge: Cambridge University Press, 2000.

Campaign Communications. In: LEDUC, Lawrence; NIEMI, Richard G.; NORRIS, Pippa

(Orgs.). Comparing Democracies 2: New Challenges in the Study of Elections and Voting. London:

Sage, 2002. p. 127-147.

. Developments in Party Communications. Washington: National Democratic Institute for International Affairs, 2005.

NORRIS, Pippa; CURTICE, John; SANDERS, David; SCAMMELL, Margaret; SEMETKO, Holli

A. On Message: Communicating the Campaign. London: Sage, 1999.

PERSE, Elizabeth M. Media Effects and Society. Mahwah: L. Erlbaum Associates, 2001.

PLASSER, Fritz; PLASSER, Gunda. Global Political Campaigning: A Worldwide Analysis of

Campaign Professionals and Their Practices. Westport: Praeger, 2002a.

La campaña Global: Los nuevos gurúes del marketing político en acción. Buenos Aires: Temas

Grupo Editorial, 2002b. 
POIRÉ, Alejandro. Retrospective Voting, Partisanship, and Loyalty in Presidential Elections: 1994. In: DOMÍNGUEZ, Jorge I.; POIRÉ, Alejandro (Orgs.). Toward Mexico's Democratization: Parties, Campaigns, Elections, and Public Opinion. London: Routledge, 1999. p. 24-56.

POPKIN, Samuel L. The Reasoning Voter: Communication and Persuasion in Presidential Campaigns. Chicago: University of Chicago Press, 1991.

SCHAFFERER, Christian (Org.). Election Campaigning in East and Southeast Asia: Globalization of Political Marketing. Aldershot: Ashgate, 2006.

SON, Young Jun; WEAVER, David H. Another Look at What Moves Public Opinion: Media Agenda Setting and Polls in the 2000 U.S. Election. International Journal of Public Opinion Research, v. 18, n. 2, p. 174-197, 2006.

SWANSON, David L.; MANCINI, Paolo (Orgs.). Politics, Media, and Modern Democracy: An International Study of Innovations in Electoral Campaigning and Their Consequences. Westport: Praeger, 1996.

TREJO DELARBRE, Raúl. Simpatía por el Rating: La Política Deslumbrada por los Medios. Ciudad del México: Cal y Arena, 2010.

VALDIVIA, Marcos; BELTRÁN, Ulises. Dilemas de estrategia: ¿'Tierra o aire? Redes sociales y decisión electoral. Política y Gobierno, número especial, p. 177-198, 2009.

VALENZUELA, Sebastián; McCOMBS, Maxwell E. Agenda-setting Effects on Vote Choice: Evidence from the 2006 Mexican Election. In: Annual Conference of the International Communication Association, 57, San Francisco, p. 24-28, 2007.

VAVRECK, Lynn. The Message Matters: The Economy and Presidential Campaigns. Princeton: Princeton University Press, 2009.

VREESE, Claes H de. Campaign Communication and Media. In: LEDUC, Lawrence, NIEMI, Richard G. y NORRIS, Pippa (Orgs.). Comparing Democracies 3: Elections and Voting in the $21^{\text {st }}$ Century. London: Sage, 2010. p. 118-140.

WALLIS, Darren. Outfoxing Leviathan: Campaigning down Mexico Way. Journal of Public Affairs, v. 1, n. 3, p. 229-238, 2001.

ZALLER, John R. The Nature and Origins of Mass Opinion. Cambridge: Cambridge University Press, 1992.

. The Myth of Massive Media Impact Revived: New Support for a Discredited Idea. In: MUTZ, Diana Carole; SNIDERMAN, Paul M; BRODY, Richard A. (Orgs.). Political Persuasion and Attitude Change. Ann Arbor: University of Michigan Press, 1996. p. 17-78.

Texto recebido em 15 de dezembro 2013. Aprovado em 12 de março de 2014. 
